\title{
LED Location Beacon System Based on Processing of Digital Images
}

\author{
Grantham K. H. Pang, Senior Member, IEEE, and Hugh H. S. Liu, Member, IEEE
}

\begin{abstract}
This paper is in the field of vehicle positioning technology for the Intelligent Transportation Systems. The ideas of an innovative light-emitting diode (LED)-based location beacon system are developed and verified. The system developed is a combination of several latest technologies which include a CMOS vision sensor, high brightness LEDs, and digital image processing techniques. It belongs to a new kind of simplex communication link. A digital camera is used to capture images contained in the LED beacon signal. The captured digital images are processed by the algorithms developed and a location code is extracted. The location code can be used for calibration of a vehicle positioning system which may consist of a GPS, Inertial Navigation System (INS) and other sensors. The issues examined include the structure of the transmitter and the receiver, the signaling method, the transmission protocol of the LED panel, the relationship between the camera capturing rate and the LED pattern update rate, the digital camera exposure technology, and the efficiency of the image processing algorithms. Experiments using a prototype transmitter and a receiver were performed. The experimental results provide a good demonstration of the viability of the ideas and methodologies developed.
\end{abstract}

Index Terms-CMOS camera, image processing, intelligent transportation systems, LED, location beacon, vehicle positioning, visible light communication.

\section{INTRODUCTION}

$\mathbf{T}$ RANSPORTATION is very important in modern human lives, which makes Intelligent Transportation Systems (ITS) very crucial in any highly urbanized city in the world. Problems such as congestion are causing substantial loss of time and money, heavy environmental pollution, high fuel consumption, and traffic accidents, and, hence, deployments in ITS are in great demand. Vehicle positioning is an enabling technology in ITS. Projects such as vehicle navigation, route planning, fleet management or any location sensitive information service depends on positioning. However, vehicle positioning is a very challenging task in cities like Hong Kong. With many high rise buildings and hilly roads, neither GPS nor dead-reckoning sensors alone can perform vehicle positioning satisfactorily.

The traffic light has been used for a long time as a traffic-signaling device, which is essential in traffic management. However, traffic light technology has remained the same over

Manuscript received April 19, 2000; revised April 24, 2001

G. Pang was with the Department of Electrical and Computer Engineering, University of Waterloo, Waterloo, ON N2L 3G1, Canada. He is now with the Department of Electrical and Electronic Engineering at The University of Hong Kong, Kowloon, Hong Kong (e-mail: gpang@eee.hku.hk).

H. Liu is with the Industrial Automation Laboratory, The University of Hong Kong, Kowloon, Hong Kong.

Publisher Item Identifier S 1524-9050(01)08262-X. the decades. Current studies [1] have shown that using light emitting diode (LED) instead of incandescent light in traffic light has many advantages including money, energy savings, and much easier maintenance. Recent research demonstrated the novel idea on the dual use of the LED-based traffic light [2]-[5]. Information can be transmitted wirelessly and optically to a photodiode-based receiver. However, there are still some problems to be solved. For example, the requirement for the line-of-sight (LOS) could limit the practical operation for LED location beacon application.

The objective of this paper is to develop the idea of intelligent traffic light, in order to increase the viability for practical usage. The aim is to develop and verify new methods for solving the line-of-sight problem in the LED beacon system. Also, new kinds of LED signal transmitter and receiver have been designed and evaluated.

\section{LITERATURE SURVEY}

\section{A. Beacon Systems and Optical Wireless}

Beacon system projects conducted worldwide include VICS and UTMS in Japan, ANTTS in Sydney and Singapore, EUROSCOUT in Europe and a beacon system in California. VICS provides drivers with real time traffic and other information through infrared, microwave beacons, and FM multiplex. UTMS is a traffic management system. Infrared beacons are used as vehicle detectors and two-way communication devices. EURO-SCOUT is an infrared beacon-based dynamic route guidance and driver information system deployed in Stuttgart and Berlin. ANTTS is a remote vehicle location system based on RF beacons. Finally, the signpost system in Huntington Beach, California is a vehicle location system based on RF beacons. It is for the effective dispatch of patrol cars during emergencies.

Kahn and Barry [8] discuss the use of infrared radiation as a medium for high-speed short-range wireless digital communication. Smyth et al. [9] review the application of optical free space link for future broadband network. Long $(0.5-5 \mathrm{~km})$ and short $(<500 \mathrm{~m})$ external and internal system are discussed. Kahn et al. [8], [10] propose the angle-diversity infrared receiver. Multiple receiving elements that are oriented in different directions are used. Using this approach, a higher optical gain and a wide FOV can be achieved simultaneously.

\section{B. LED Traffic Light}

Incandescent lamps have been used in traffic lights for several decades. With the advancement in technologies, high brightness LEDs are available which provide very good reasons to substitute the incandescent light. LED features high brightness, 
TABLE I

COMPARISONS BETWEEN INCANDESCENT LAMPS AND LEDS FOR THE TRAFFIC LIGHT APPLICATION [1], [11]

\begin{tabular}{|c|c|c|}
\hline & $\begin{array}{l}\text { Incandescent } \\
\text { lamps }\end{array}$ & LEDs \\
\hline Technology & $\begin{array}{l}\text { Voltage applied } \\
\text { across tungsten in } \\
\text { a glass sphere } \\
\text { cause heating }\end{array}$ & $\begin{array}{l}\text { Electron energy } \\
\text { state transition } \\
\text { across a p-n } \\
\text { junction }\end{array}$ \\
\hline $\begin{array}{l}\text { Power } \\
\text { Consumption } \\
\text { for a } 12 \text {-inch } \\
\text { light } \\
\end{array}$ & 150 Watts & 14 Watts \\
\hline $\begin{array}{l}\text { Life } \\
\text { expectancy }\end{array}$ & $\begin{array}{l}\text { Replaced twice a } \\
\text { year }\end{array}$ & $\begin{array}{l}\text { Very long, and } 10- \\
15 \text { years guaranteed. }\end{array}$ \\
\hline $\begin{array}{l}\text { External light } \\
\text { filter }\end{array}$ & $\begin{array}{l}\text { Filter is needed to } \\
\text { generate colored } \\
\text { light }\end{array}$ & No filter is needed \\
\hline $\begin{array}{l}\text { Shock } \\
\text { resistance }\end{array}$ & Less & High \\
\hline Light reflector & Needed & No needed \\
\hline $\begin{array}{l}\text { Failure } \\
\text { Consequence }\end{array}$ & Sudden signal out & $\begin{array}{l}\text { Only a portion of } \\
\text { the LED panel fail. }\end{array}$ \\
\hline
\end{tabular}

low energy consumption, long durability, and the availability of red, green, amber, and even other colors. In the U.S., Philadelphia has taken the lead in deploying the LED signal head technology [1]. Twenty-eight thousand red signals in the city are replaced with an estimated annual cost savings of US\$1.2 million. The savings come from the reduction of energy consumption, less lamp replacement, and reduced potential liability for personal injury. Ibrahim and Beasley [11] describe the benefit of replacing the incandescent lamps with LEDs in the traffic lights in London. Pilot test sites were planned in 1998 in the Victoria area in London. Estimated total annual saving is $£ 1.4$ million sterling if all the traffic lights in London are to be replaced by LEDs. Table I shows a comparison between incandescent lamps and LEDs in their usage in traffic lights.

Besides the above advantages of using LED in traffic lights as a signaling device, another important feature of LED is that it has fast switching capability. By fast switching on and off, the LED can transmit information with the visible light in a wireless way. This is similar to an infrared LED for optical wireless communication. While infrared LED operates at 780-950 nm, a red light LED operates at around $626 \mathrm{~nm}$ [12].

Pang et al. [2]-[7] describe the idea, design, evaluation and implementation of LED wireless communication systems. Both analog audio broadcast and digital data communication systems are covered. An intelligent traffic light was fabricated and evaluation experiments were conducted. The viewing angle (half power) is found to be $30^{\circ}$. For digital signal, the transmission rate can reach $500 \mathrm{~kb} / \mathrm{s}$ and the throughput is around 2000 bytes/s. For an optical power at the receiver of $0.8 \mu \mathrm{W}$, the bit error rate (BER) is around $9 \times 10^{-8}$. The specification of the intelligent LED traffic light is shown in Table II. The traffic light developed is shown in Fig. 1.

Although using LEDs in visible band for wireless optical data transmission is interesting and potentially very useful, there is still much room for research and improvement. The developed LED communication system can be categorized as a directed LOS system [6]. This is due to the properties of the red LEDs
TABLE II

PROPERTIES OF AN INTELLIGENT LED TRAFFIC LIGHT.

\begin{tabular}{l|l}
\hline Signal color & Red \\
\hline Construction & $\begin{array}{l}441 \text { red ultra-bright LEDs } \\
\text { with a luminous intensity } \\
\text { of 2000 mcd @20mA }\end{array}$ \\
\hline Diameter & 8 inches \\
\hline Nominal usage & $17 \mathrm{~V} ; 2 \mathrm{~A}$ \\
\hline $\begin{array}{l}\text { Nominal power } \\
\text { consumption }\end{array}$ & $34 \mathrm{~W}$ \\
\hline $\begin{array}{l}\text { Luminous intensity } \\
\text { Viewing angle (half } \\
\text { power) }\end{array}$ & $300 \mathrm{~cd}$ \\
$\begin{array}{l}\text { Distance for audio } \\
\text { transmission (outdoors) }\end{array}$ & $30^{\circ}$ \\
\hline
\end{tabular}

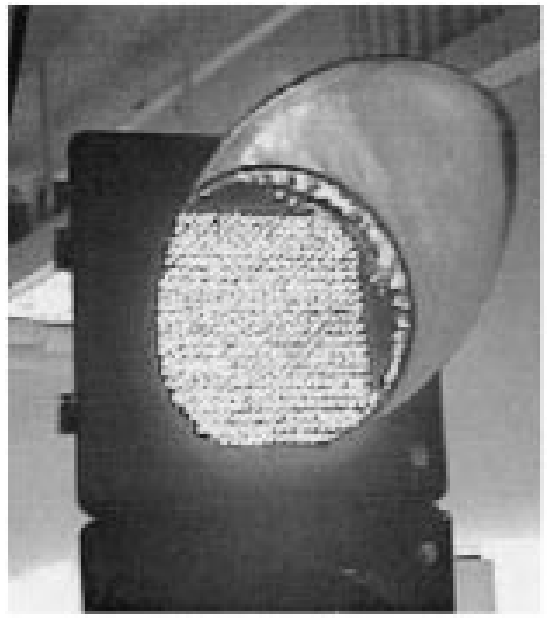

Fig. 1. Intelligent LED traffic light capable of transmitting digital and analog data.

and the photodiode-based receiver design. This limits the field of view (FOV) or the viewing angle for the receiver. Hence, the idea of using a digital camera as a receiver is developed in this paper. Image processing technique is used to extract the information from an intelligent LED traffic light.

\section{PRoblem DEFINITION AND ANALYSIS}

\section{A. Digital Camera as an LED Beacon Signal Receiver}

Data communication using wireless visible light LED signal is possible. Previous research has used photodiode to sense the visible light signal. However, there is a significant LOS problem. If the receiver is displaced by a small angle, say a few degrees, the signal may not be received. In this paper, a digital camera is proposed for reducing the LOS requirement. Nevertheless, some problems should be overcome when a camera-based receiver is used for sensing the LED visible light signal. The problems are stated in the followings.

1) Capturing Frame Rate: LED can be switched on and off at a fast rate that human eye cannot detect. The upper limit of switching rate depends on the structure and materials inside the LED. For an off-the-shelf video-conferencing camera, the capturing frame rate is usually 30 frames per second. By using the transmission protocol presented in Section VII of this paper, the capturing rate of the digital camera for the beacon 


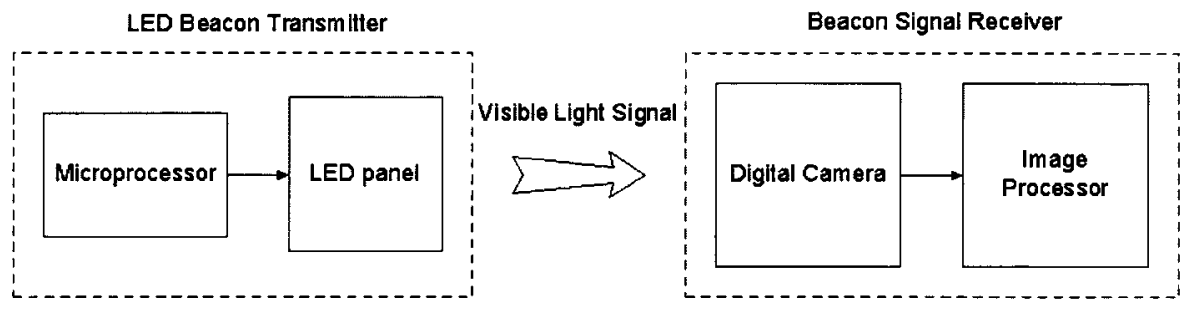

Fig. 2. A block diagram of the LED location beacon system.

should be more than 400 frames per second. Thus an ordinary video-conferencing camera is not suitable. Although there exists some high frame rate digital camers for fast motion analysis, these systems are usually very costly and bulky. Thus, a specific camera is needed and a protocol should be devised in order to strike a balance between cost and performance.

2) Communication Protocols: A suitable protocol for the LED beacon communication is needed. Otherwise, the digital camera can only receive frames of image without meaning. Also, the camera frame rate and the LED flashing frequency are not assumed to be synchronized. The communication protocol should be able to handle the unsynchronized nature of the signal reception.

3) Exposure Technology: Unlike a film-based camera which expose the whole image at the same time, a CMOS camera exposes a number of pixel rows at a time. This technology is known as the electronic rolling shutter (ERS). This works well for capturing normal motion items and events. However, when capturing very fast changing items such as the flashing LEDs, the LED may flash several times during one frame period. This may cause undesirable transitional patterns to be captured. Thus, suitable methodologies are needed to enable the reception of LED data by a CMOS camera.

4) Image Processing for the LED Beacon Signal: Image processing could be a time consuming job for extracting the location code from the LED beacon signal. If a car is passing by the LED beacon at maximum allowable speed on the street, then the time available for processing the received LED image may be short. It is assumed that the receiver does not know whether there is a LED beacon in front of it. Even if there is a LED beacon in the captured image, the position of the beacon in the image is not known. Thus, the image processing method should handle the signal head detection task. Moreover, both the vehicle and the surrounding things are moving on the road. Due to time lag between successive captured images, the extraction of the LED data may be affected depending on the image processing algorithm. Thus, methods for reducing the effect of movements on the data extraction performance are needed.

\section{B. Methodology in the Proposed Research}

Fig. 2 shows a block diagram of the LED location beacon system based on image processing.

An effective image processing algorithm is needed to decode the location information from the beacon signal. A microprocessor is used to control the switching of the LED panel according to the devised transmission protocol. The LED beacon system can be in the form of a traffic light. Thus, it becomes

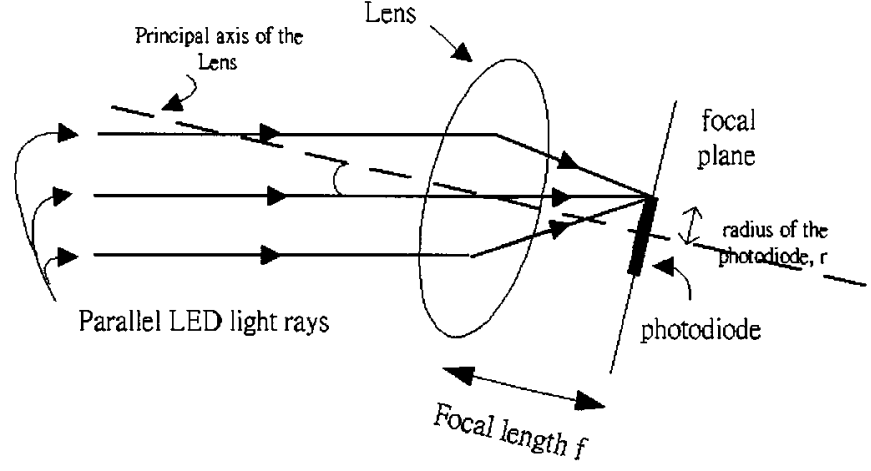

Fig. 3. Assumed parallel LED light rays with incident angle $\theta_{\text {scmi }}$ converged at the focal plane and just fall on the photodiode.

both a traffic signaling system and a location beacon. A digital camera is used to capture the image sequence of the LED beacon. These images are then processed for extracting the location information.

\section{ANALYSIS OF THE LOS PROBLEM}

\section{A. Photodiode-Based Receiver Issues}

A lens is used to concentrate the power of the LED signal to the photodiode. Let $\theta_{\text {semi }}$ be the semiangle of the receiver, $f$ be the focal length of the lens and $r$ be the radius of the photodiode (assumed to be circular).

Fig. 3 shows how large the angular movement of the receiver is permitted without disrupting the communication when the LED traffic light and the receiver are properly aligned. The light rays in the figure are just converged at the edge of the photodiode. If the incident angles of the LED light rays are bigger than $\theta_{\text {semi }}$, then no signal can be received. The FOV or semiangle of the receiver is thus given by the following:

$$
\theta_{\text {semi }}=\tan ^{-1}\left(\frac{r}{f}\right)
$$

In an experiment, the FOV of the LED receiver was found to be $5^{\circ}$. The radius of the photodiode used in the LED receiver, $r$, is $4 \mathrm{~mm}$. The focal length of the lens is $50 \mathrm{~mm}$. Thus by (1), $\theta_{\text {semi }}=4.57^{\circ}$ which is close to the experimental result.

\section{B. Digital Camera as a Receiver}

A digital camera provides a wider FOV LED beacon receiver which is low-cost and small in size. Besides receiving the LED signal, a digital camera can serve other useful purposes such as traffic condition detection, collision avoidance, rear viewer, automatic vehicle, lane keeping, etc. The focal length of the CMOS digital camera in the prototype OmniVision OV5017 


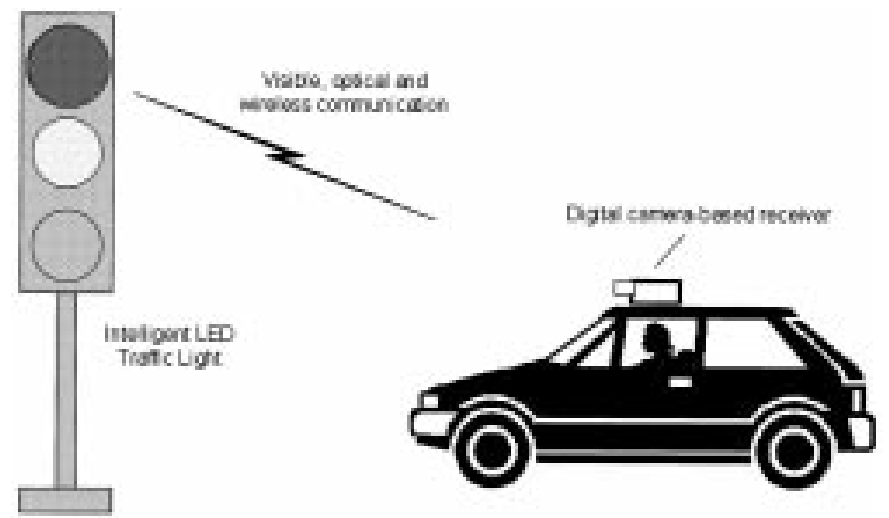

Fig. 4. A scenario of the proposed LED location beacon system based on a digital camera receiver.
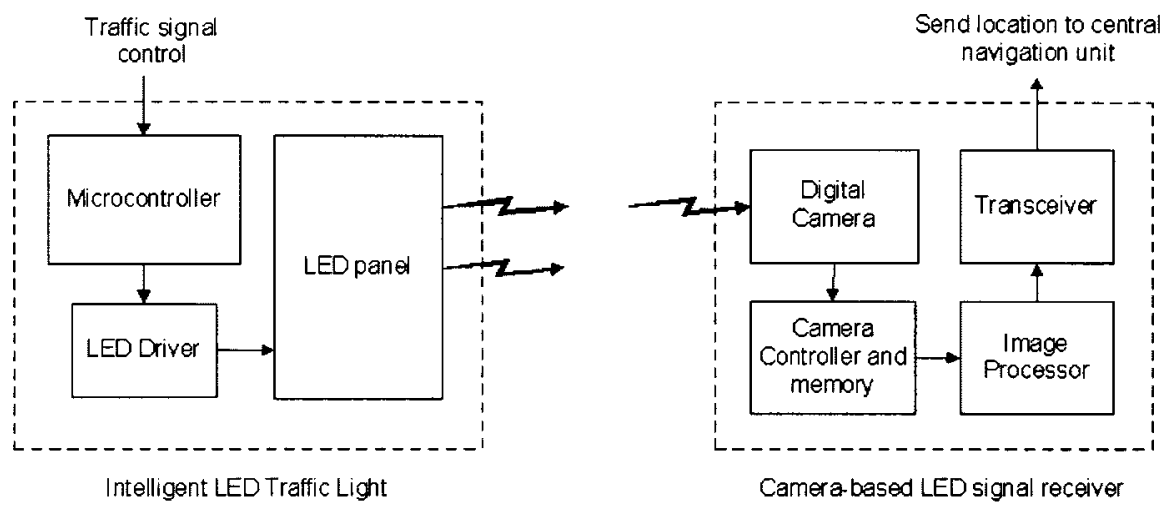

Camera-based LED signal receiver

Fig. 5. System architecture of the proposed location beacon system.

[13] is $7.4 \mathrm{~mm}$. It has a sensor width of $4.2 \mathrm{~mm}$. Thus, by (1), the horizontal FOV (semiangle) of the camera is $29.6^{\circ}$. This is close to the $31.7^{\circ}$ specified in the camera specification.

\section{SYSTEM DESCRIPTION}

The developed location beacon system consists of an intelligent LED traffic light and a camera-based receiver. A location code is transmitted by the intelligent LED traffic light. A passing-by vehicle equipped with the camera-based receiver can obtain this location code through this visible optical wireless link. The scenario is shown in Fig. 4.

The system architecture is shown in Fig. 5. At the transmitter side, the three major components are microcontroller, LED driver, and LED panel. The receiver consists of a digital camera, a camera controller, memory units for storing images, an image processor, and a transceiver for external communication.

\section{A. Intelligent LED Traffic Light}

When a photodiode-based receiver is used, all the LEDs in a panel are flashed on and off at the same time. When this camera-based receiver is used, the LED panel is divided into partitions. Within each partition, the LED is turned on and off simultaneously. Hence, different patterns can be shown on the LED panel.

A microcontroller which contains a unique location code mainly performs two tasks. First, it is responsible for controlling the ON-OFF of the different partitions. Thus, different patterns on the LED panel are actually flashing at a rate which too fast for the human eye to detect. The sequence of flashes is in accordance with a proposed protocol which is described later. The second function of the microcontroller is to receive the traffic light control signal from the traffic control device. Thus, the intelligent traffic light also functions normally as a traffic signaling device.

Driver circuits are necessary to switch the array of LEDs on and off rapidly, while providing enough current to the LEDs. A power MOSFET can be used in the intelligent LED traffic light design. Fig. 6 shows a diagrammatic illustration of the intelligent LED traffic light.

\section{B. Digital-Camera-Based LED Signal Receiver}

The location beacon receiver consists of a digital camera, a camera controller, memories for storing images, an image processor, and a transceiver for external communication. Fig. 7 shows the structure of the digital camera-based LED signal receiver.

A CMOS digital camera module is used to capture the images of the intelligent LED traffic light. The operation of the digital camera is controlled by the control unit for image capturing, reading, parameter modification, and monitoring. The captured images are stored in the memory units and then processed. The image processor extracts the location code of the LED traffic light from the images by the processing algorithms developed. The transceiver is used for sending out the extracted location code to a main navigation unit. 


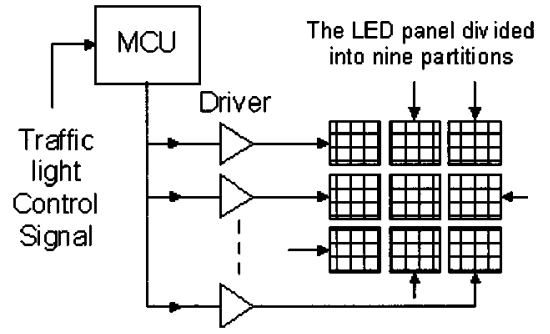

Fig. 6. The architecture of the intelligent LED traffic light for a camera-based receiver.

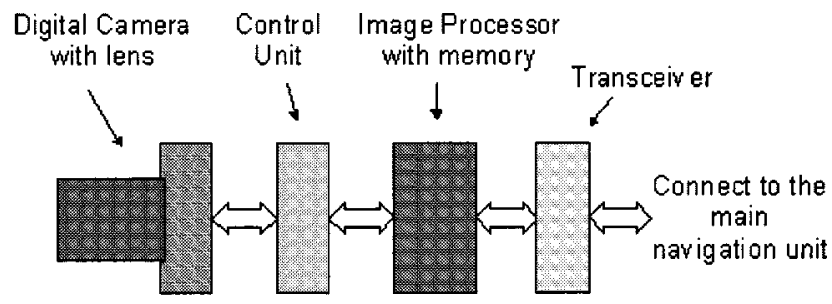

Fig. 7. The structure of the digital camera-based LED signal receiver.

\section{SignALing Method of the INTELLIGENT LED TRAFFIC LIGHT}

In the LED panel used for the photodiode-based receiver, all the LEDs in the panel flash simultaneously. However, for the proposed camera-based receiver, the LED panel is divided into nine partitions which is shown in Fig. 8. More panel partitions (16 or 25) will give a larger channel capacity. The only drawback is that it would be more difficult to identify the panel pattern in the subsequent image processing stage due to poorer resolution.

Within each partition, all the LEDs are either on or off. In a specific period, some partitions are on and some are off, and the LED panel shows a specific pattern. Four examples of the LED panel are illustrated in Fig. 9.

Each unique pattern and its complementing pattern are used to represent a location code. The principle is just like pasting a bar code on a signpost which tells its location. The location code is shown on the intelligent LED traffic light but is not visible to the human eyes. The patterns of the LED panel are changed at a fast rate according to a protocol. Thus, for a driver or a pedestrian, the traffic light signal appears to be constantly illuminated. With a digital camera receiver, the encoded LED pattern which represents a unique location can be captured. Although the LED panel is partitioned into nine regions which generates 512 different patterns, only part of them can be used for signaling. The reason is related to the protocol and decoding methods which are described in the later sections.

\section{TRANSMISSION PROTOCOL}

The design of the transmission protocol for the intelligent LED traffic light is based on the differencing of image sequences, which can be used to extract the location code. A good transmission protocol can lead to an efficient and simple system. The criteria of the protocol design, the proposed A-On-A'-Off protocol and the method of transmitting more than one digit are described in this section.

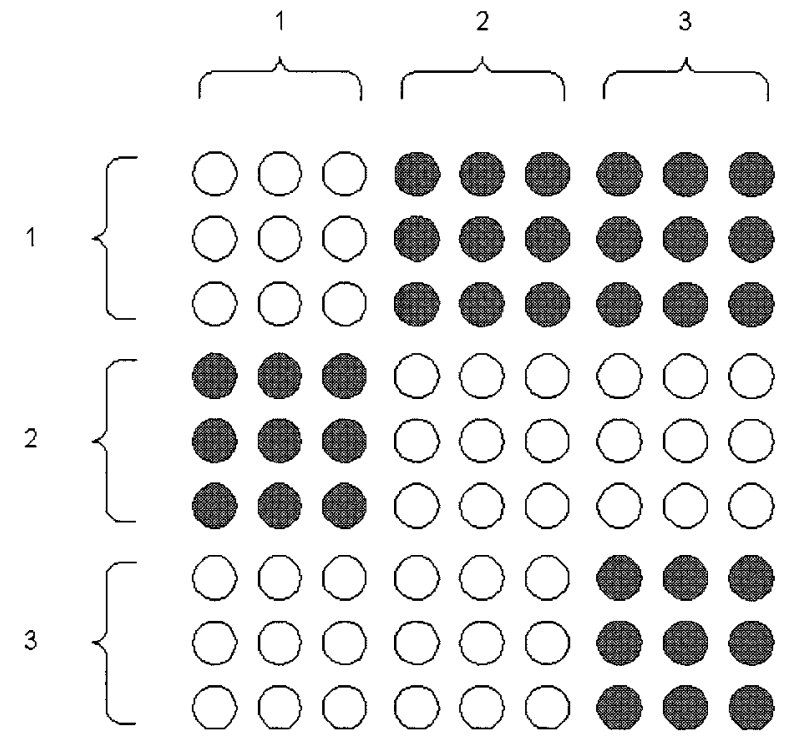

Fig. 8. The LED panel divided into nine partitions.
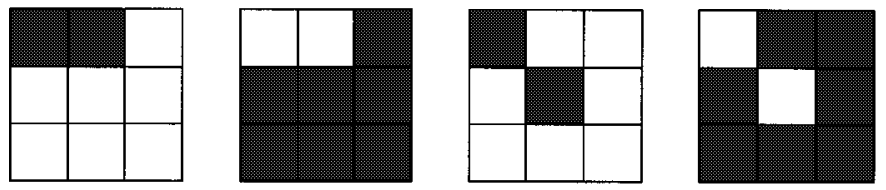

Fig. 9. Four examples of the LED panel patterns.

\section{A. Criteria for the Transmission Protocol Design}

The following have to be considered in the design of the transmission protocol:

- properties of the LED Traffic Light transmitter;

- properties of the digital camera-based receiver;

- nature of the communication link.

The intelligent LED traffic light acts as a signaling device for traffic control, as well as a location beacon to transmit position information to the vehicles. There are some requirements on the design of the transmission protocol. Firstly, the LED panel should be capable of transmitting information wirelessly and optically. The light emitted by the LED panel should appear to be constant (with undetectable flashing), so that data transmission is not noticeable to the eyes. Secondly, the captured images are stored into the memory units for processing. The size of an digital image is often large, thus, the transmission protocol should minimize the number of image frames required for the extraction of a location code. This leads to the saving in the use of memory units and a decrease in the execution time. Thirdly, the location beacon is a simplex communication link. The protocol is designed to be simple and workable. Thus, the protocol design should prevent the need for synchronization between the transmitter and the receiver. This means that a vehicle can receive a location code at any time it approaches the intelligent LED traffic light.

\section{B. A-On-A'-Off Protocol}

A-On- $\mathrm{A}^{\prime}$-Off protocol is proposed to satisfy the requirements stated in last subsection. The terms are defined first. A represents a particular LED pattern which is illustrated in Fig. 10, 


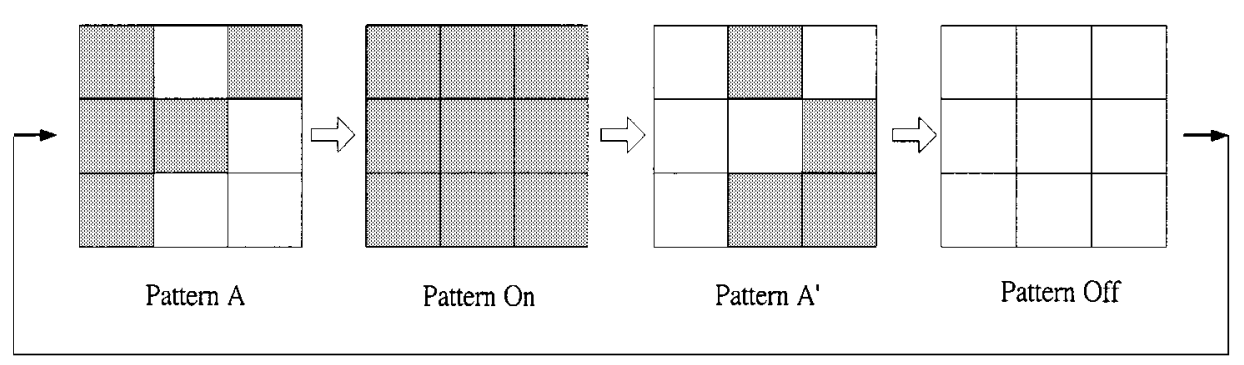

Fig. 10. Pattern transmission sequence using the A-On- $\mathrm{A}^{\prime}$-Off protocol.

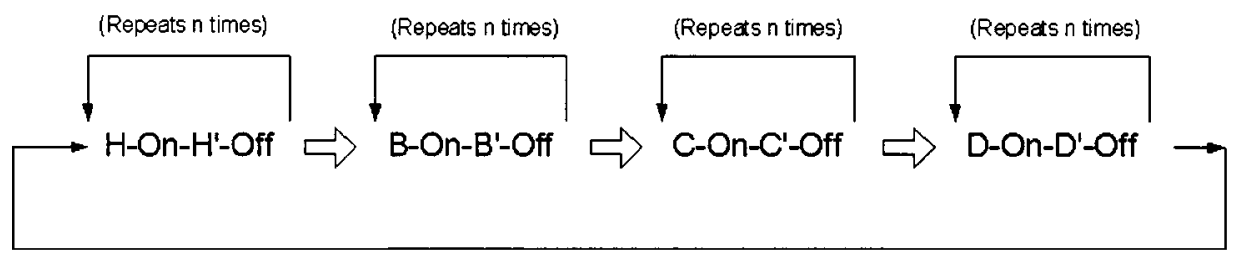

Fig. 11. Example of a transmission protocol sending a 3-digit location code.

TABLE III

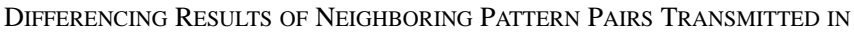
A-ON-A'-OFF PROTOCOL

\begin{tabular}{l|c}
$\begin{array}{l}\text { Neighboring Pattern } \\
\text { Pairs }\end{array}$ & $\begin{array}{l}\text { Differencing } \\
\text { Results }\end{array}$ \\
\hline A - On & $A^{\prime}$ \\
\hline On - $A^{\prime}$ & $A$ \\
\hline$A^{\prime}-$ Off & $A^{\prime}$ \\
\hline Off - A & $A$ \\
\hline
\end{tabular}

while $\mathrm{A}^{\prime}$ denotes the complement of pattern $\mathrm{A}$. On means that all the partitions are turned on and Off means that all the partitions are turned off.

Using the A-On-A'-Off protocol, the LED patterns A, On, $\mathrm{A}^{\prime}$ and Off are displayed sequentially for a short period. This sequence is repeated periodically. With fast switching between these patterns, the LED panel is perceived to be on at the same brightness. This is due to the adaptation of the human vision system. Thus, the A-On-A'-Off protocol satisfies the constant brightness requirement.

The differencing of the LED patterns transmitted in the A-On-A'-Off protocol satisfies the other requirements. The resulted pattern obtained from the differencing of neighboring pattern pairs can be summarized in Table III.

From Table III, it can be observed that the (absolute of) differencing of neighboring pattern pairs always yields pattern $\mathrm{A}$ and $\mathrm{A}^{\prime}$. If pattern $\mathrm{A}$ and $\mathrm{A}^{\prime}$ is assigned to a unique location code, then a differencing process of a received pair of images can yield the location code of an intelligent LED traffic light. Hence, the camera only needs to capture two consecutive images for the differencing process. This minimizes the size of the memory unit. Moreover, no synchronization between the transmitter and the receiver is needed.

Let $t_{\text {LED_pat }}$ be the duration of showing a particular LED pattern. Hence, the LED pattern update rate $f_{\text {LED_pat }}$ is

$$
f_{\text {LED_pat }}=1 / t_{\text {LED_pat }} \text {. }
$$

Let $f_{\mathrm{LED}}$ be the resulted flashing rate of the LED panel. Referring to Fig. 10, each partition on the LED panel is observed to be turned on and off for the duration of $2 * t_{\text {LED_pat }}$ in a A-On-A'-Off cycle. Thus the resulted flashing period for each LED partition is $4 * t_{\text {LED_pat }}$ and

$$
f_{\text {LED }}=1 / 4 * t_{\text {LED_pat }} \text {. }
$$

For the LED panel to appear constantly illuminating, the minimum $f_{\text {LED }}$ should be $50 \mathrm{~Hz}$. Hence, the LED pattern update rate $f_{\text {LED_pat }}$, by (2) and (3), should be $4 * 50 \mathrm{~Hz}=200 \mathrm{~Hz}$.

\section{Transmitting More Than One Digit}

The intelligent LED traffic light can transmit several digits or even a stream of information to a digital camera-based receiver. It will be shown in a later subsection that a LED panel with 9 partitions can present 61 different location codes. Thus a reduced set of printable ASCII character (including A-Z, 0-9, and other symbols) can be represented by a LED pattern. A message can be transmitted by the intelligent LED traffic light if the transmission protocol can handle more than one digit. Also, transmitting more digits can increase the number of unique location code. In fact, the A-On-A'-Off protocol can be modified in order to support the transmission of several digits. As illustrated in Fig. 11, $\mathrm{H}$ denotes a header pattern. The $\mathrm{B}, \mathrm{C}$, and $\mathrm{D}$ are three different patterns that represents the first, second, and third digit of the location code, respectively.

\section{Digital CAMERA Frame RATE AND DATA RECEIVING}

It is desired that when two neighboring image frames are differenced, a location code can be found as shown in Table III. In practice, differencing on two consecutive frames captured by the digital camera receiver usually cannot yield the location code. This is due to the capturing of transitional pattern (the combination of two different LED patterns) and the relative frequency difference of LED pattern update and the camera capturing rate. Thus, in this section, the relationship between the frequency of LED pattern update rate and the camera capturing rate is presented. It can be shown that a location code must be found by two capturing actions without the need of any synchronization between the transmitter and the camera. 


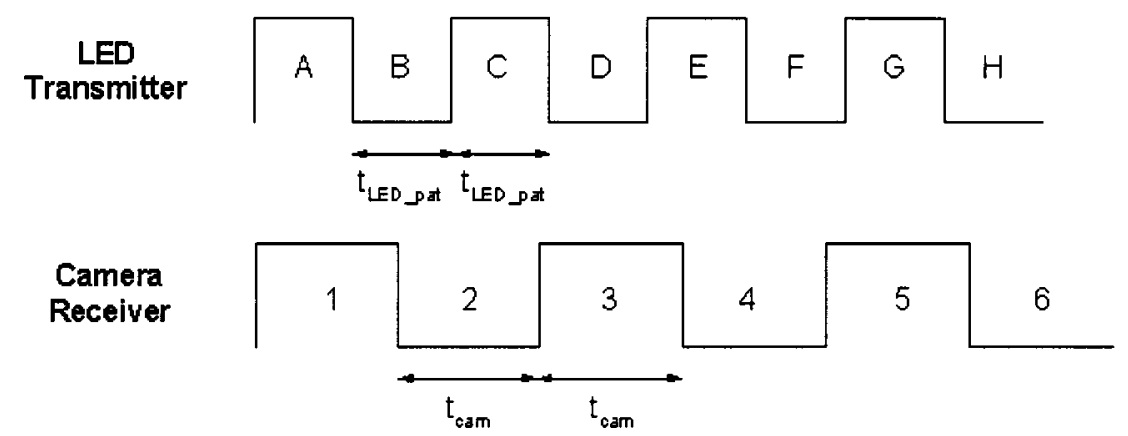

Fig. 12. Timing relationship between the LED transmitter and the camera receiver when $t_{\text {LED_pat }}<t_{\text {cam }}$.

Four images are obtained and processed in each capturing action. The transmitter is using the A-On- $\mathrm{A}^{\prime}$-Off protocol. The four frames captured is targeted to be T1, G1, T2, and G2 where $\mathrm{T} 1$ and $\mathrm{T} 2$ are transitional frame which are not useful and G1 and $\mathrm{G} 2$ are two different and consecutive good patterns which are useful for location code extraction. Here, the consecutive patterns means the consecutive patterns from the A-On- $\mathrm{A}^{\prime}-\mathrm{Off}$ sequence such as A-On and $\mathrm{A}^{\prime}$-Off. Six codes are generated from the differenced pairs in each capturing action and only one of them is the location code. However, a second capturing action that yield the same location code is needed to select the location code from the nonuseful codes.

The notations needed in this section are defined:

- $f_{\text {cam }}$ capturing rate of the digital camera;

- $t_{\text {cam }}$ period of an image frame captured by the digital camera;

- $f_{\text {LED_pat }}$ pattern update rate of the LED panel;

- $t_{\text {LED_pat }}$ duration of showing a particular pattern on the LED panel;

- $t_{\mathrm{exp}}$ exposure time of an image frame of the digital camera

$$
f_{\text {cam }}=1 / t_{\text {cam }} \text {. }
$$

The digital camera is assumed to expose the whole frame of image at the same time. Thus, the transitional pattern (combination of different patterns) is just the overlapping of the different patterns. Thus, the transitional pattern is useful for location code extraction. However, some CMOS digital cameras are using an exposure technology called ERS. There is an exposure starting time difference between each pair of neighboring rows in the image. This may cause unusable transitional patterns. The ERS and the camera shuttering issues are described in a later section.

\section{A. Camera Exposure Time Equals the Frame Period}

The focus of this section is on the analysis of the system when $t_{\exp }=t_{\text {cam }}$. This analysis can give a clearer idea of how the relationship between $f_{\text {cam }}$ and $f_{\text {LED_pat }}$ can affect the certainty of getting the location code. Moreover, this case is possible in real situation. The analysis is divided into three cases which are shown as the following:

- $t_{\text {cam }}>t_{\text {LED_pat }}$

- $t_{\text {cam }}=t_{\text {LED_pat }}$

- $t_{\text {cam }}<t_{\text {LED_pat. }}$.

These cases are described in the following subsections.
TABLE IV

IMAGe CAPTURED BY THE Digital CAMERA When $t_{\text {LeD_pat }}<t_{\text {camn }}$.

\begin{tabular}{l|l|l|l|l|l}
\hline $\begin{array}{l}\text { Frame } \\
\text { Number }\end{array}$ & 1 & 2 & 3 & 4 & 5 \\
\hline $\begin{array}{l}\text { Captured } \\
\text { pattern }\end{array}$ & $\mathrm{A} / \mathrm{B}$ & $\mathrm{B} / \mathrm{C}$ & $\mathrm{C} / \mathrm{D} / \mathrm{E}$ & $\mathrm{E} / \mathrm{F}$ & $\mathrm{F} / \mathrm{G} / \mathrm{H}$ \\
\hline
\end{tabular}

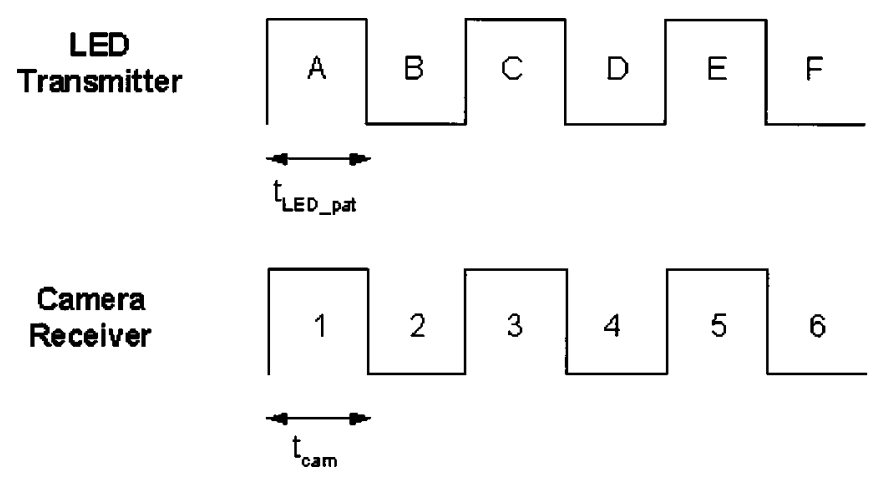

Fig. 13. Timing relationship between the LED transmitter and the camera receiver when $t_{\text {cam }}=t_{\text {LED_pat }}$ and in phase.

1) $t_{\text {cam }}>t_{\text {LED_pat }}$ : The timing relationship between the LED transmitter and the camera receiver is shown in Fig. 12. It can be seen in Fig. 12 that every image captured by the digital camera is a transitional pattern which is shown in Table IV.

Comments: In case 1), it is not possible for proper communication because the transitional patterns (combination of two or more different LED pattern) are always captured by the digital camera. Thus the location code cannot be extracted by the differencing of the image pairs.

2) $t_{\text {cam }}=t_{\text {LED_pat }}:$ The timing of the LED and the camera are aligned which is shown in Fig. 13.

Comments: Only when the timing of the LED transmitter and the camera receiver are exactly aligned (in phase), the image captured by the camera is useful for finding the location code. However, the chance for this situation is very rare.

3) $t_{\text {cam }} \leq t_{\text {LED_pat }}$ : In this case, the captured image by the digital camera is useful for location code finding. Useful LED patterns can be captured besides the transitional patterns. The following analysis derives the conditions to ensure that a location code must be generated from two capturing actions. The timing relationship between the LED transmitter and the camera receiver when $t_{\text {cam }} \leq t_{\text {LED_pat }_{\text {_p }}}$ is shown in Fig. 14 . 


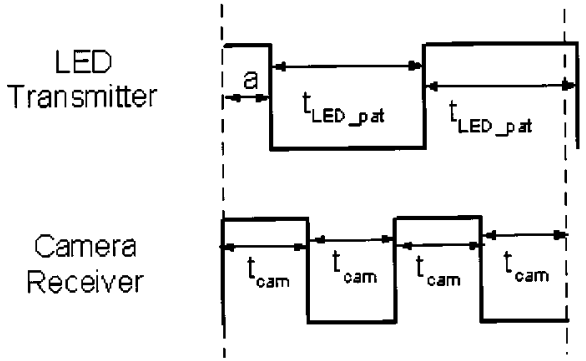

Fig. 14. The timing relationship between the LED transmitter and the camera receiver when $t_{\text {cam }} \leq t_{\text {LED_pat. }}$.

Assumes that ' $a$ ' is the phase difference between the LED pattern update rate and the camera capturing rate. Moreover, the following condition is assumed:

$$
0 \leq a \leq t_{\text {cam }}
$$

Assuming the first frame captured is a transitional frame which is shown in Fig. 14. The following conditions are needed for ensuring a location code to be decoded:

1) a good frame captured in the second frame;

2) a transitional frame be captured in the third frame;

3) a good frame captured in the fourth frame.

For requirement 1)

$$
2 t_{\text {cam }} \leq t_{\text {LED_pat }}+a .
$$

For requirement 2)

$$
t_{\text {LED_pat }}+a \leq 3 t_{\text {cam }} \text {. }
$$

For requirement 3)

$$
4 t_{\text {cam }} \leq 2 t_{\text {LED_pat }}+a .
$$

By (5) and (7)

or

$$
t_{\text {LED_pat }}+a \leq 3 t_{\text {cam }} \Rightarrow t_{\text {LED_pat }} \leq 3 t_{\text {cam }}
$$

$$
\frac{1}{3} t_{\text {LED_pat }} \leq t_{\text {cam }} \text {. }
$$

By (5) and (8),

$$
\begin{aligned}
4 t_{\text {cam }} & \leq 2 t_{\text {LED_pat }}+a \Rightarrow 4 t_{\text {cam }} \leq 2 t_{\text {LED_pat }}+t_{\text {cam }} \\
3 t_{\text {cam }} & \leq 2 t_{\text {LED_pat }} \\
t_{\text {cam }} & \leq \frac{2}{3} t_{\text {LED_pat. }} .
\end{aligned}
$$

By (5) and (6),

$$
\begin{aligned}
2 t_{\text {cam }} & \leq t_{\text {LED_pat }_{\text {_pa }}}+a \Rightarrow 2 t_{\text {cam }} \leq t_{\text {LED_pat }}+t_{\text {cam }} \\
t_{\text {cam }} & \leq t_{\text {LED_pat }}
\end{aligned}
$$

which confirms the requirement in this case.

Thus, by (9) and (10), the condition which ensures a location code to be found from two capturing actions (patterns T1, G1, $\mathrm{T} 2$, and G2 captured in each capturing action) is

$$
\frac{1}{3} t_{\text {LED_pat }} \leq t_{\text {cam }} \leq \frac{2}{3} t_{\text {LED_pat }} .
$$

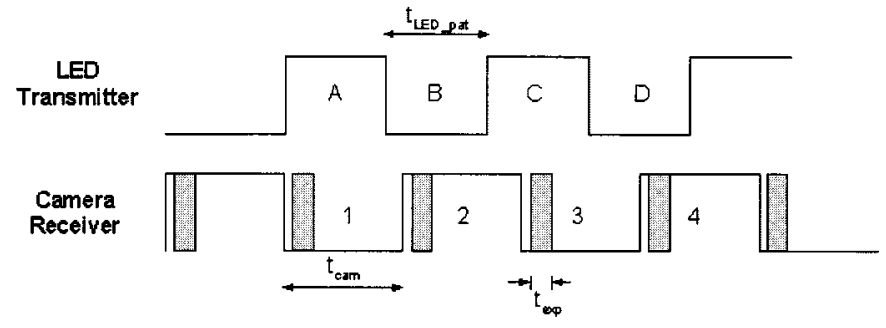

Fig. 15. The timing relationship between the LED transmitter and the camera receiver when the camera exposure time is shorter than the frame period.

\section{B. Camera Exposure Time Shorter Than the Frame Period}

Fig. 15 shows the timing relationship between the LED transmitter and the camera receiver when the camera exposure time is shorter than the frame period.

The position of the exposure window within a camera frame period depends on the vertical position of the LED panel in the image.

When $t_{\text {cam }} \leq t_{\text {LED_pat }}$, the same arguments in Section A apply here. Two capturing actions guarantee a location code will be found. However, the chance of obtaining a location code in one capturing action is higher, because the chance of getting a transitional frame is less. Moreover, for $t_{\text {cam }}>t_{\text {LED_pat, }}$, it is possible to get a location code which is different from the case described in Section VIII-A. In general, the following requirements favor the extraction of a location code in four captured frames (referring to Fig. 15)

- The ratio $t_{\text {exp }} / t_{\text {LED_pat }}$ should be much smaller than 1 , say, 0.01 . The smaller the ratio, the less probable a transitional pattern are captured within the four frames.

- $t_{\text {cam }} \leq t_{\text {LED_pat }}$ to avoid getting the same pattern within the four frames.

It should be noted that when the ratio of $t_{\text {cam }} / t_{\text {LED_pat }}$ is above 1 , the LED pattern update rate is faster than the camera capture rate (as shown in Fig. 16). In this figure, $t_{\text {cam }} / t_{\text {LED_pat }}$ is 5 which means that the LED changes patterns five times faster than the camera capturing rate. However, the requirement (a) should be observed, which means that the camera exposure time should be smaller than the LED pattern update rate and much smaller than the frame period of the camera.

The experiments in this paper were based on $t_{\text {cam }} / t_{\text {LED_pat }}=5$. It enables the use of a digital camera with a frame rate of only one-fifth of the LED pattern update rate.

\section{Effect of Camera Shuttering}

There are two kinds of camera shuttering technologies which affect the LED pattern captured. The first kind is whole frame exposing which is just like the method used in a film-based analog camera. The whole image frame is exposed at the same time by the same duration. ERS is usually employed in CMOS cameras. The idea of the ERS is illustrated in Fig. 17.

There are two pointers to control the exposure time of an image: reset pointer and read pointer. Initially, all rows of the imager are inactivated. When the capturing action starts, the reset pointer begins to activate the rows and goes downward. The staying period of the reset pointer in one row equals the row processing time. After activation of a specified number of 


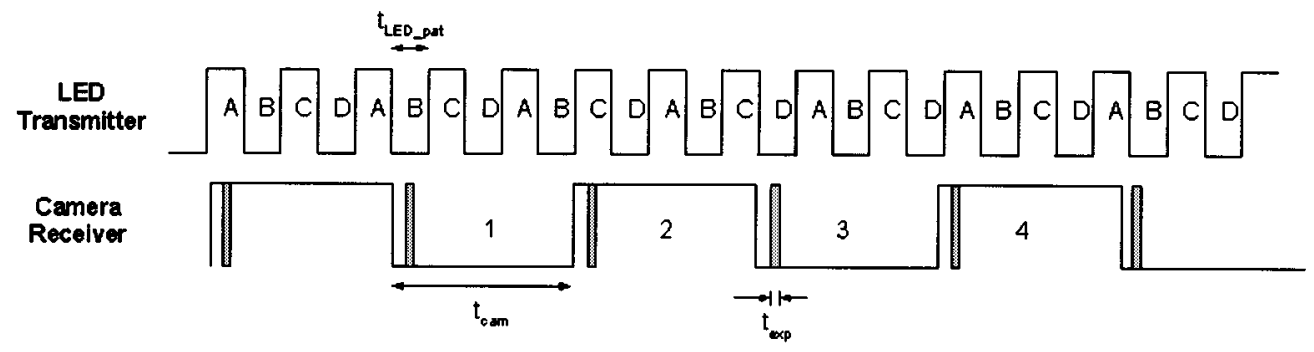

Fig. 16. The timing relationship between the LED transmitter and the camera receiver when the ratio is 5 .

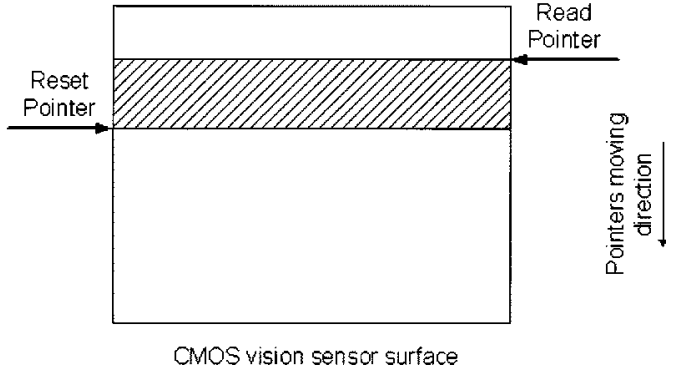

Fig. 17. Illustration of the Electronics Rolling Shutter.

rows (in a duration of integration time which is set by the user), the read pointer starts rolling off and transfers the exposed row image to an analog-to-digital converter row by row. The staying period of the read pointer in one row is also the row processing time. In other words, there is an exposure starting time difference between each pair of neighboring rows in the image. The exposure time difference between the first and the $n$th row is equal to ( $n *$ row processing time).

For a whole frame exposing camera, the transitional LED pattern captured is usually usable for the location code extraction. But for an ERS camera, most of the transitional LED patterns captured are not useful.

\section{RECOVERY OF LOCATION CODE From the CAPTURED IMAGES}

After the images are captured by the digital camera and stored in the memory unit, the location data is extracted from them. Fig. 18 shows a flowchart for the recovery of a location code from the captured images by using the image processing algorithms developed.

\section{A. Differencing}

The differencing process is used for detecting whether there is any signal within the images captured by the digital camera. If there is a (LED) location beacon signal, this process extracts it from the sequence of images. It works by eliminating the common background within a pair of images and retains only the flashing beacon signal. The differencing process includes the subtraction, absoluting, and noise reduction (shown in Fig. 19).

Although the differencing process is a fast and simple method for detecting and extracting the portion of the LED panel within the images, it is affected by the movement of other vehicles in view and its own movement. Two methods are proposed to

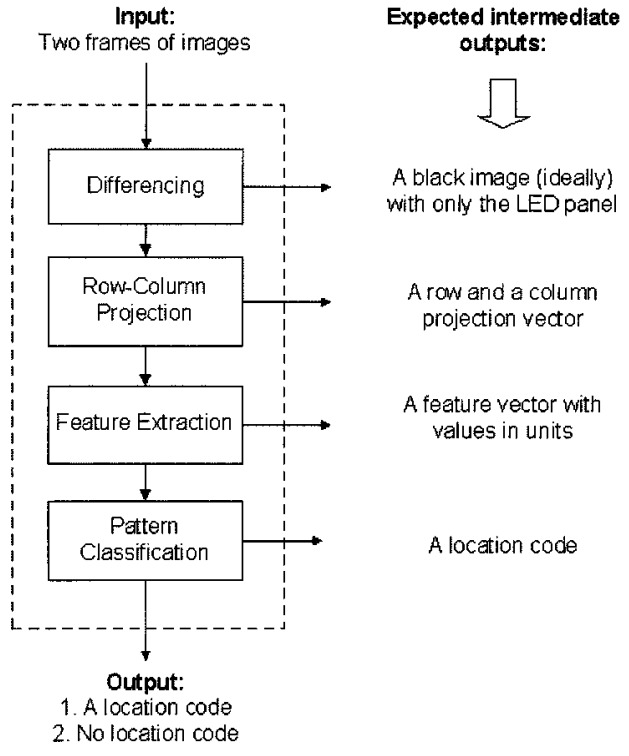

Fig. 18. Flowchart for the recovery of a location code from the captured images by using the image processing algorithms developed.

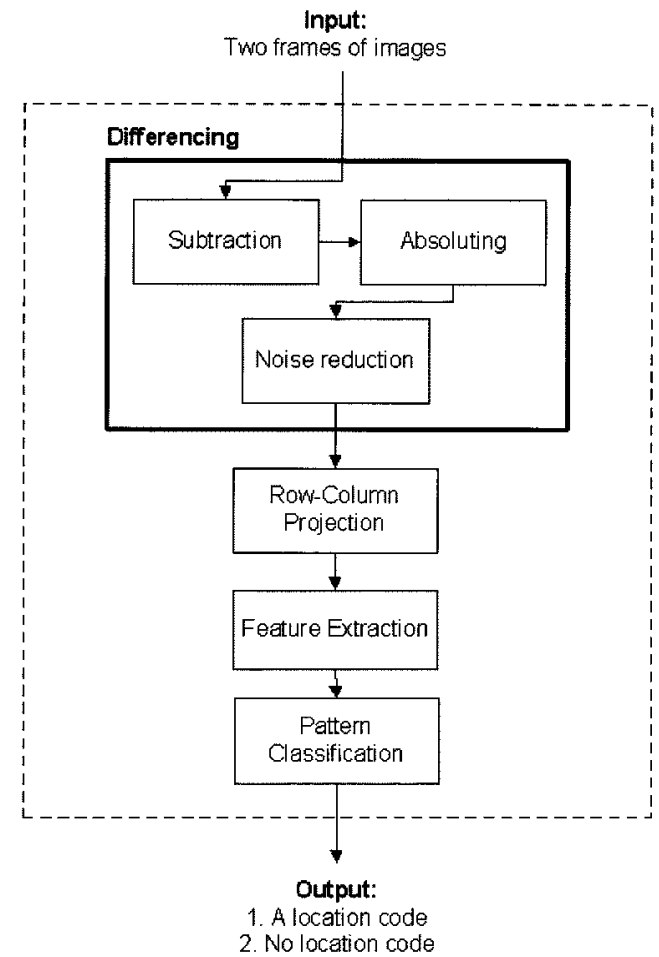

Fig. 19. The differencing processing in the image processing algorithm. 


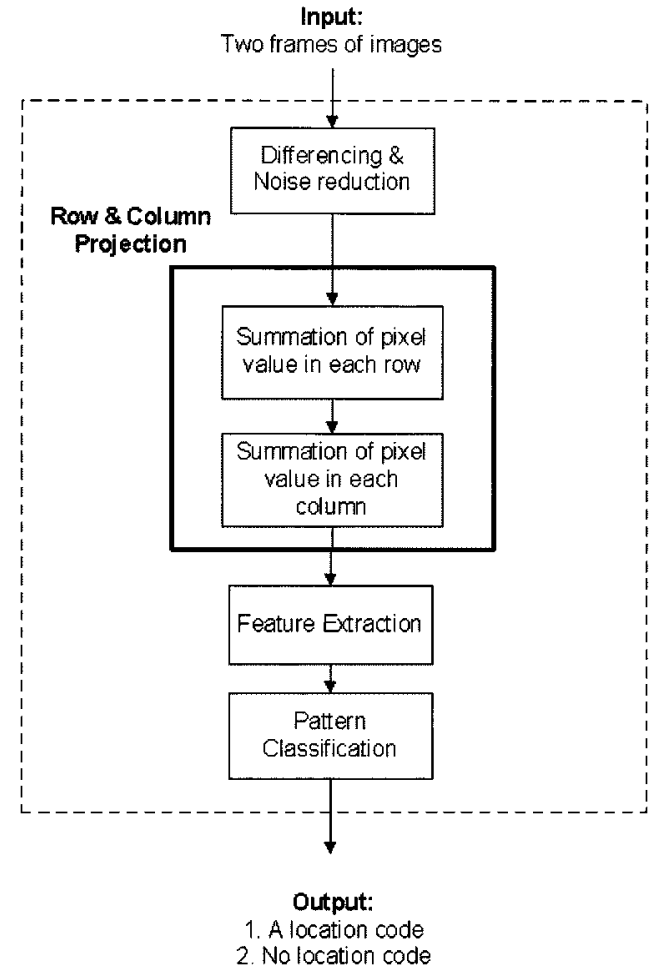

Fig. 20. The row and column projection process.

tackle the problem of noise when differencing due to the movement of objects in the background and the camera movement. These methods give a better differenced image for the row and column projection process. The first method is to use a median filter and a thresholding process. The second method involves the use of a red light filter before the camera lens. Moreover, the region of interest of the digital camera can be selected such that the area outside can be excluded.

\section{B. Row and Column Projection}

After the differencing process, the resulted image is used for row and column projection. The purpose of this procedure is to convert the 2-D differenced image into a 1-D vector to facilitate the feature extraction process. All pixel values in each row are summed and stored in a vector which is called a row projection vector. The column projection vector is found by summing the pixels in each column. The process in the image processing algorithm is shown in Fig. 20.

An example of the row and column projection process is shown in Fig. 21. Let $h$ be an integer value showing the height of a projection peak. The resulted row projection vector contains the equal number of elements to the height of the differenced image. The dimension of the column projection vector equals the width of the differenced image.

\section{Feature Extraction}

After getting the row and column projection vectors, the peaks of projection values in the two vectors are found. The ratio of these peak values are then calculated, rounded off to the nearest integers, and reduced to units. The projection values

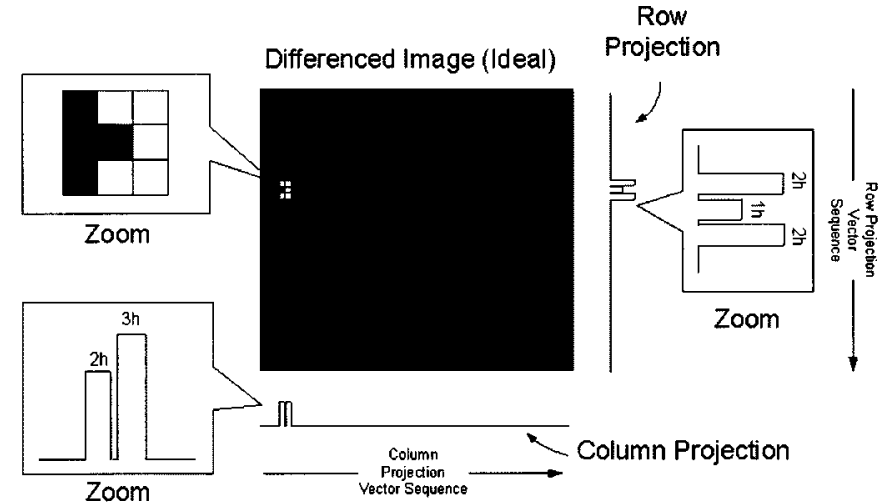

Fig. 21. Row and column projection process.

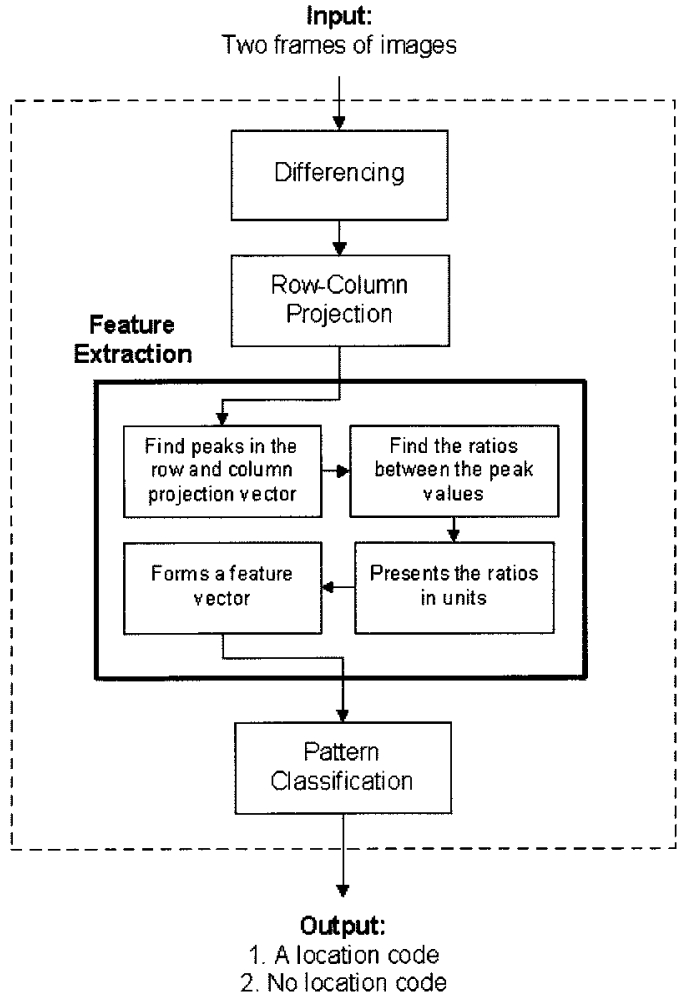

Fig. 22. The feature extraction process.

other than the peaks are ideally zero. These ratios (in units) of the peaks found in the two projection vectors are combined to form a single feature vector. The number of elements in the feature vector ranges from $0-6$ if a $3 \times 3$ LED panel is used. This feature vector is mapped to a location code. The feature extraction processing in the image process algorithm is shown in Fig. 22.

For example, the feature vector for the pattern in Fig. 21 should be [ $\left.\begin{array}{lllll}2 & 1 & 2 & 2 & 3\end{array}\right]$ which represents a unique location code. Elements from top to down in the row projection and then left to right in the column projection are processed and gives the feature vector. In an actual situation, the row and column projections waveform may not be so precise due to noise and distortion of the LED panel signal. Thus, a robust algorithm for finding the peaks and the corresponding values in the differenced image is needed in order to provide correct results. 


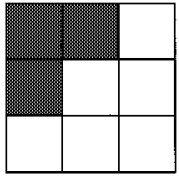

(a)

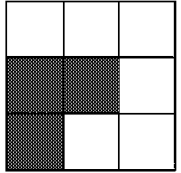

(b)

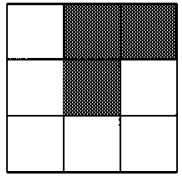

(c)

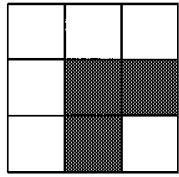

(d)
Fig. 23. Four different LED patterns having the same feature vector.

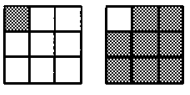

4

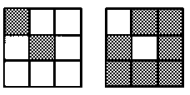

7

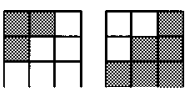

2

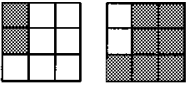

5

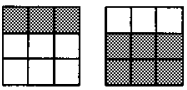

8

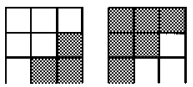

3

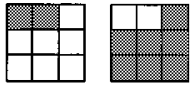

6

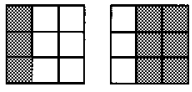

9

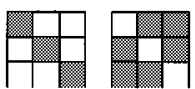

Fig. 24. LED patterns and their complement ( 9 out of 61 pairs) used to present the location codes.

TABLE V

FeATURE VeCtors OF THE LED PATTERN-COMPLEMENT PAIRS.

\begin{tabular}{l|l|l}
\hline & \multicolumn{2}{|l}{ Feature Vector } \\
\hline Code & Normal & Complement \\
\hline 1 & 11 & 233233 \\
\hline 2 & 112 & 223133 \\
\hline 3 & 211 & 133223 \\
\hline 4 & 1111 & 223223 \\
\hline 5 & 3111 & 33222 \\
\hline 6 & 1113 & 22233 \\
\hline 7 & 2121 & 123123 \\
\hline 8 & 1212 & 321321 \\
\hline 9 & 111111 & 222222 \\
\hline
\end{tabular}

1) Feature Vectors for the $3 \times 3$ LED Panel: For a $3 \times 3$ LED panel, there should be 512 different patterns. However, not all of them can be used for representing a location code. This is due to the nature of the row and column projection and the feature extraction process. The reason is illustrated in Fig. 23.

The four different LED patterns have the same feature vector [ $\left.\begin{array}{llll}2 & 1 & 2 & 1\end{array}\right]$, and can represent just one location code. Thus, the number of usable pattern is reduced. Moreover, a pattern and its complement pattern are both assigned the same unique location code due to the design of the transmission protocol. This further reduce the number of location codes which can be presented by the LED panel. Finally, only 61 pairs of the LED patterns with their complements are used to represent the location codes. Nine of the LED patterns and their complements are shown in Fig. 24.

The feature vectors representing the nine LED pattern-complement pairs (in Fig. 24) are shown in Table V.

The number of location code which can be presented by the LED panel can be increased by sending a multidigit code as mentioned in Section VII.C. However, the multidigit code cannot contain identical neighboring digit due to the transmission protocol design. Also, there should be a digit reserved for the header. Thus, for a 3-digit location code, the number of location code supported should be $60 \times 59 \times 59=208860$.

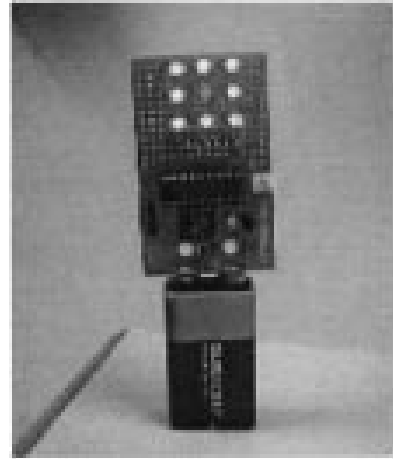

Fig. 25. The 8-LED transmitter prototype (constantly illuminating and transmitting information).

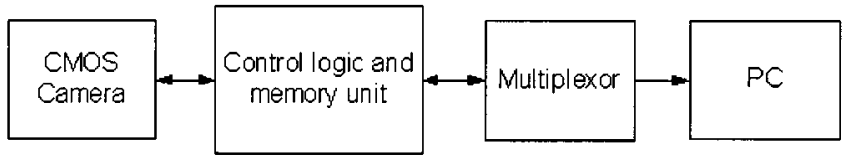

Fig. 26. Block diagram of the digital camera receiver prototype.
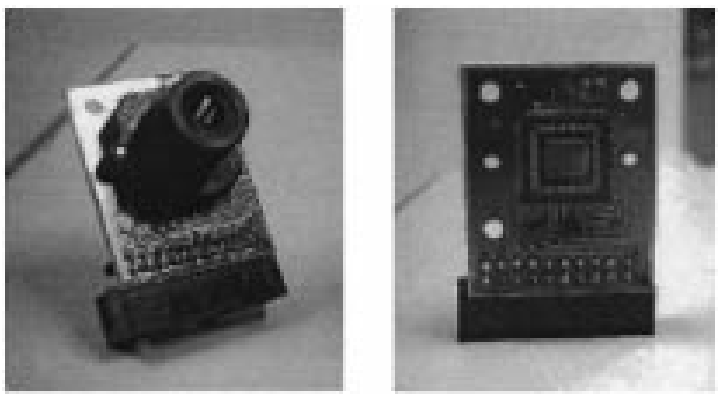

Fig. 27. The OmniVision CMOS camera module (with and without a lens) employed in the beacon receiver.

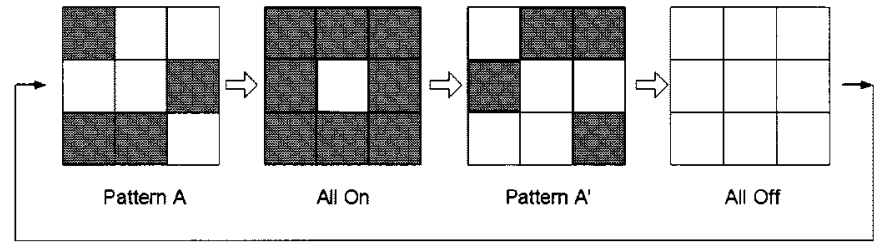

Fig. 28. The LED patterns transmitted with the A-On- $\mathrm{A}^{\prime}$-Off protocol in this set of experiments.

\section{Pattern Classification}

Once the feature vector is obtained, the corresponding location code can be found by searching a table relating the feature vectors and the location codes. For a fast search, the feature vector data can be organized into a tree structure with three branches (for value 1,2, and 3 in the feature vector). For a $3 \times 3$ LED panel, the feature vector for a location code digit contains 0 to 6 elements. Thus, the tree structure contains 6 levels. Searching algorithm such as heuristic search and bi-directional search can be used.

\section{EXPERIMENT AND Results FOR the BEACON System}

In this section, two major sets of experiments for simulating data transmission from an LED traffic signal are presented. The 


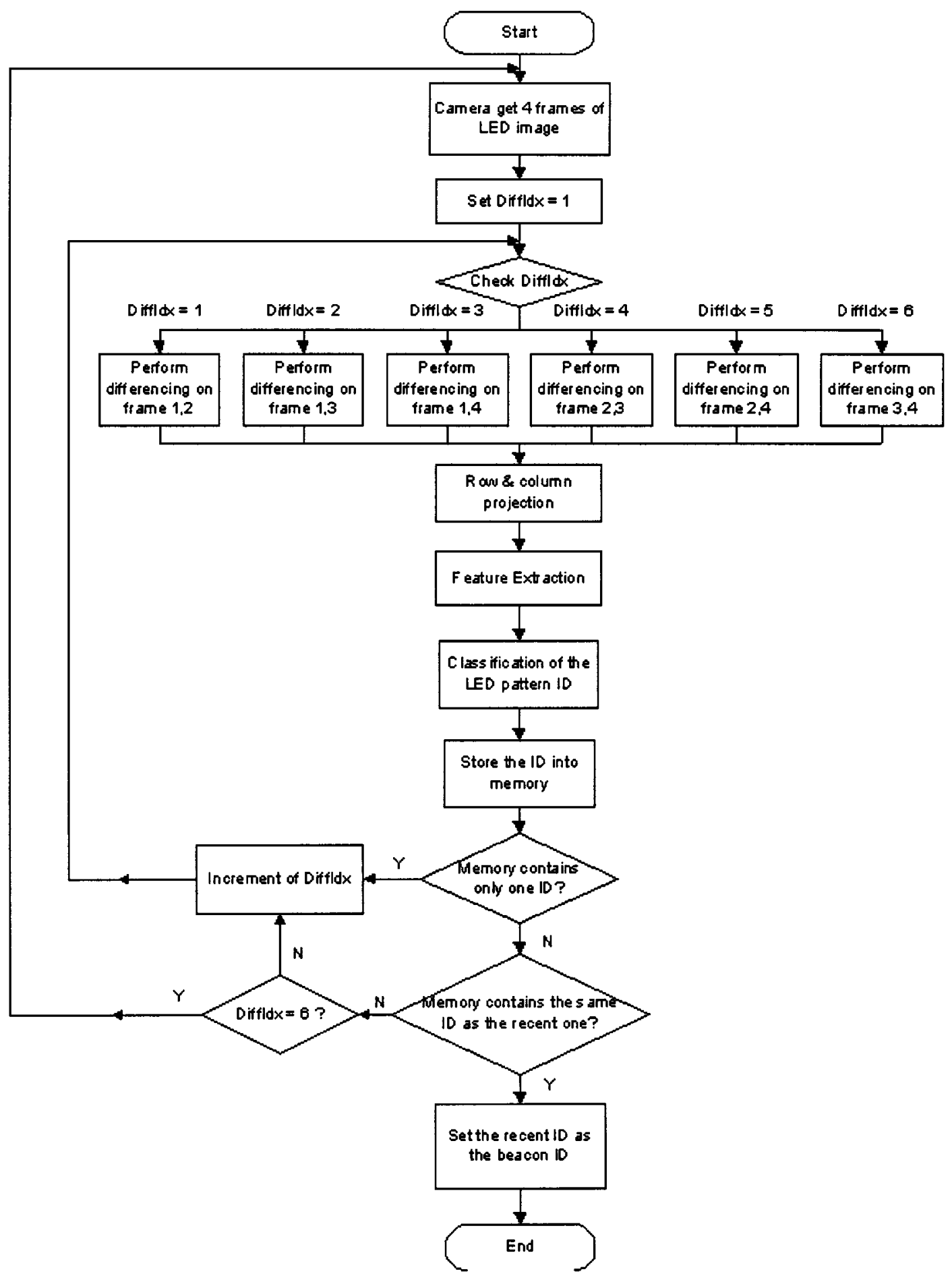

Fig. 29. The flowchart of the main program for extracting the location code from four images captured with the digital camera receiver prototype.

first set is for demonstrating an LED beacon system based on image processing. The second part is on noise reduction in the differencing process.

\section{A. Experiments on Obtaining a Location Code}

1) Transmitter and Receiver Prototypes:

a) LED transmitter prototype: A prototype LED transmitter panel consisting of eight LEDs (the center LED is not used) is used to transmit data. LED patterns are updated on the panel in a fast way such that a constant illumination is seen by human eyes. By flashing the LED patterns in the A-On- $\mathrm{A}^{\prime}$-Off protocol, a location code is transmitted by the LED panel. The transmitter prototype consists of a microcontroller (a BASIC stamp) and eight LEDs. Only eight LEDs are used because the microcontroller has only eight output pins for control. The A-On- $\mathrm{A}^{\prime}$-Off protocol is programmed in the EEPROM of the microcontroller. It commands the LEDs to transmit the patterns with a pattern update rate of $200 \mathrm{~Hz}$. The LED panel is seen 
to be constant illuminating. Fig. 25 shows the LED transmitter developed for the experiments.

b) Digital camera receiver prototype: The digital camera receiver can capture and store 4 frames of $384 \times 288$ gray scale image from the digital camera each time. The 4 images stored in the memory unit can be transferred to a PC through the printer port. A PC is used to control the camera receiver prototype. It can start the image capturing action, modify and monitor the parameters of the digital camera such as the exposure time and the sensitivity. The prototype receiver consists of a digital camera, a control logic unit, a memory unit, a multiplexor, and a PC. The structure of the camera receiver is shown in Fig. 26.

The OmniVision OV5017 CMOS imager [13] is used in this experiment set. The photographs of the camera module with and without a lens are shown in Fig. 27.

2) Experimental Procedures: The LED panel was set to flash at the patterns as shown in Fig. 28 using the A-On- $\mathrm{A}^{\prime}$-Off protocol developed.

The eight LEDs represent eight partitions in the intelligent LED traffic light. The LED panel is constantly illuminating while transmitting the information. The digital camera receiver is connected to a PC that controls the operation and gets image data from it. Four frames of 8-bit gray scale image with size $384 \times 288$ pixel are captured into the PC from the digital camera. The capturing rate of the digital camera is $50 \mathrm{f} / \mathrm{s}$ and the shuttering technology employed is ERS.

Both the receiver and the transmitter are kept stationary during the experiment. The four captured images are processed with the methods described in Section IX. The image processing algorithms are implemented in MATLAB 5.0. The main program flow chart is shown in Fig. 29. Six possible combinations of the differencing process are performed. The row and column projections of the differenced images are found. The feature extraction of the projection curve is performed. Then the location code represented by the feature vector is yielded. A location is found provided that two identical location codes are obtained from the six differenced images. This is to remedy the problem of getting a transitional pattern within the four captured images.

For the feature extraction process from the column projection vector, the highest peak in the column projection curve is found first. Then the values of the two peaks on the left of the highest peak are found, if any exist. After this, the values of the two peaks on the right of the highest peak are found, if any exist. The ratios of the peak values are found and are reduced to units. After the same process for the row projection curve, the feature vector is yielded.

3) Experimental Results: The four frames captured are shown in Fig. 30. The ERS shuttering method is used in the digital camera receiver. Thus, the transitional image captured by the camera is not useful for finding the location code. Frame 3 in Fig. 30 is a transitional pattern. The algorithm is written to adapt to this effect by checking for two identical location code within the six differenced images.

The differenced image and the corresponding row and column projection curves are shown in Fig. 31. The zoomed differenced image and the corresponding row and column projection curves are shown in Fig. 32.

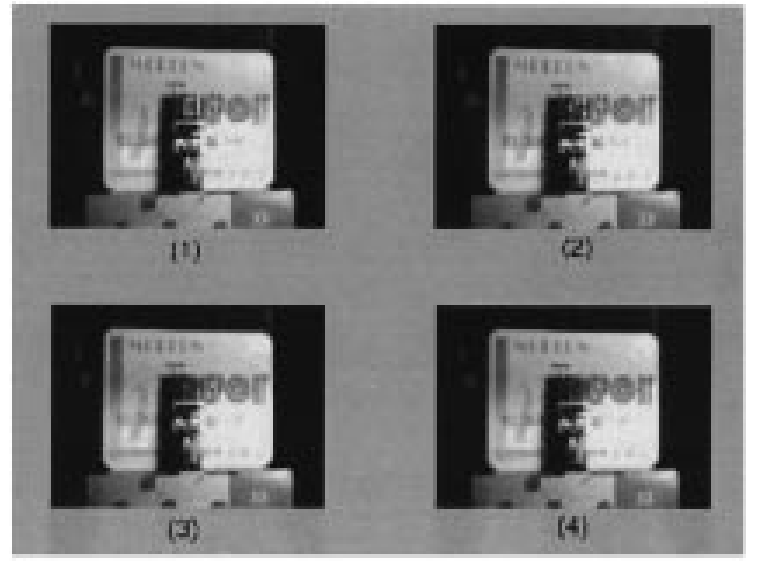

Fig. 30. Four images captured under a brighter background.

TABLE VI

FEATURE VeCtORS OF THE Six DifFERENCED IMAGE IN THE EXPERIMENT WITH BRIGHTER BACKGROUND.

\begin{tabular}{|c|c|c|c|}
\hline $\begin{array}{l}\text { Differencing } \\
\text { of frames }\end{array}$ & $\begin{array}{l}\text { Feature } \\
\text { Vector } \\
\text { number }\end{array}$ & $\begin{array}{l}\text { Feature } \\
\text { Vector }\end{array}$ & $\begin{array}{l}\text { Location } \\
\text { Code } \\
\text { Obtained }\end{array}$ \\
\hline $1-2$ & 1 & {$\left[\begin{array}{llllll}1 & 1 & 2 & 2 & 1 & 1\end{array}\right]$} & Yes \\
\hline $1-3$ & 2 & {$\left[\begin{array}{llllll}3 & 2 & 2 & 3 & 2 & 2\end{array}\right]$} & No \\
\hline $1-4$ & 3 & {$\left[\begin{array}{llllll}3 & 2 & 3 & 3 & 2 & 3\end{array}\right]$} & No \\
\hline $2-3$ & 4 & $\left.\begin{array}{l|llllll} & {[} & 1 & 1 & 1 & 1\end{array}\right]$ & No \\
\hline $2-4$ & 5 & {$\left[\begin{array}{lllllll}2 & 1 & 1 & 1 & 1 & 2\end{array}\right]$} & Yes \\
\hline $3-4$ & 6 & {$\left[\begin{array}{llll}1 & 0 & 0 & 0\end{array}\right]$} & No \\
\hline
\end{tabular}

A thresholding procedure with index 0.3 (ranges from $0-1$ ) is used to convert the differenced image to black and white before the row and column projection process. This is to reduce the noise after differencing. It also reduces the values of the projection curve after the row and column projection process which may save storage resource if embedded processor is used for implementing the data extraction process. The feature vectors of the differenced image pairs found by the program developed are shown in Table VI.

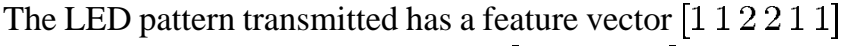

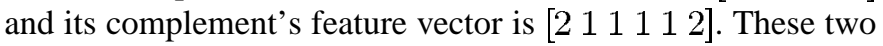
feature vectors are assigned to a location code. If both of these two vectors are found within the 6 differenced images, then the location code can be obtained. Thus, by Table VI, the location code can be retrieved from the four captured images because feature vector 1 and 5 match the desired feature vectors.

\section{B. Experiments on Noise Reduction in the Differencing Process}

The purpose of these experiments is to reduce the noise in the differenced image due to the background objects movements and the camera movement between the time of capturing two frames of image. The thresholding and median filtering process are employed. The results are shown to be dependent on the size of the LED signal in the image and the extent of the noise due to the object and camera movements.

The median filter is useful for removing isolated noises. The size of the noise to be removed depends on the radius of the filter. However, median filtering may also filter out 


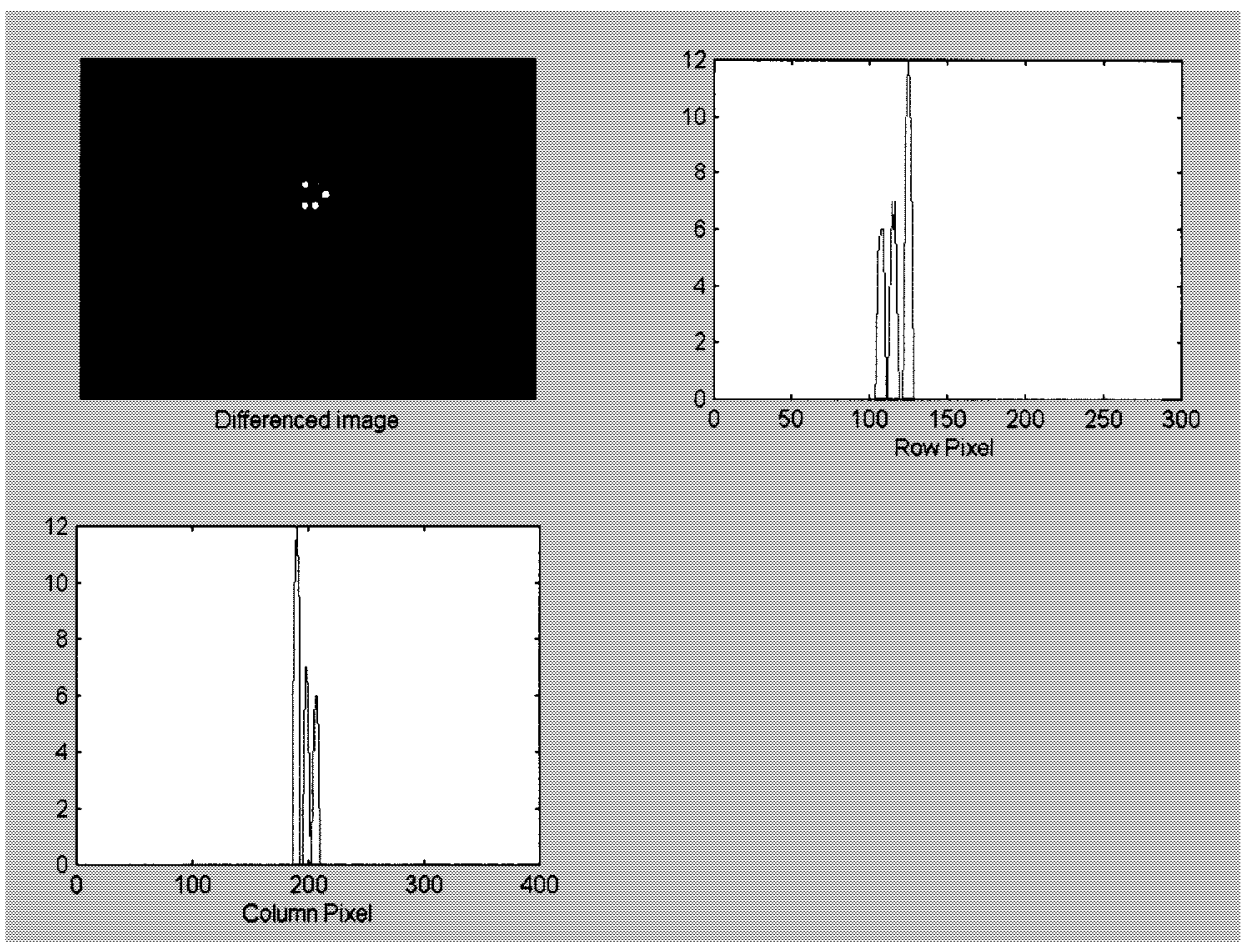

Fig. 31. Differenced image of frame 1 and 2 with the row and column projections.

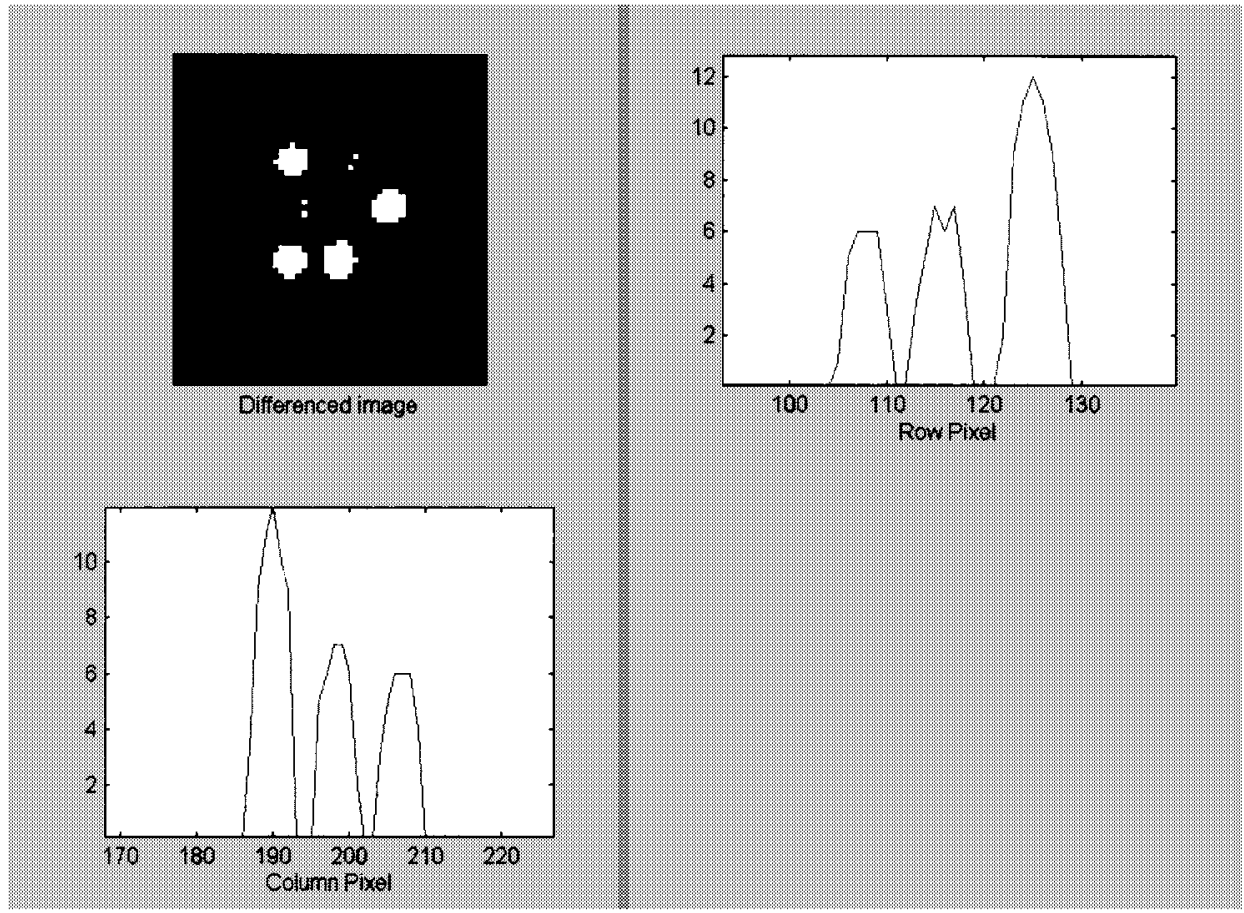

Fig. 32. Zoom-in of the differenced image of frame 1 and 2 with the row and column projections.

the LED signal partitions. Thus, only the median filter with a small radius can be used for removing limited amount of noises. The thresholding process changes a gray level image into a black and white one according to a thresholding index. This helps to remove the noise with intensity smaller than the LED signal.

Fig. 33 shows an image containing a traffic light with a simulated pattern X. Fig. 34 is an image similar to Fig. 33 but with the LED pattern all-on and the image shifted to the right by 2 pixels. The difference between Figs. 33 and 34 are shown in Fig. 35 which shows the complement of pattern $X$ with background noises due to the shift.

A thresholding process with index 160 (of 256) and three times of median filtering with radius of one pixel are applied to Fig. 35 in order to filter the background noises. The result is shown in Fig. 36. 


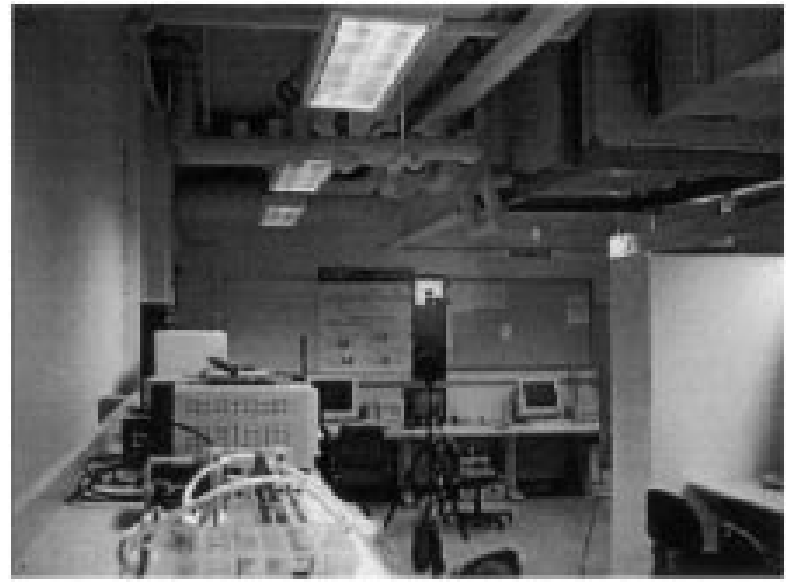

Fig. 33. Image with a LED Traffic light showing a pattern $X$.

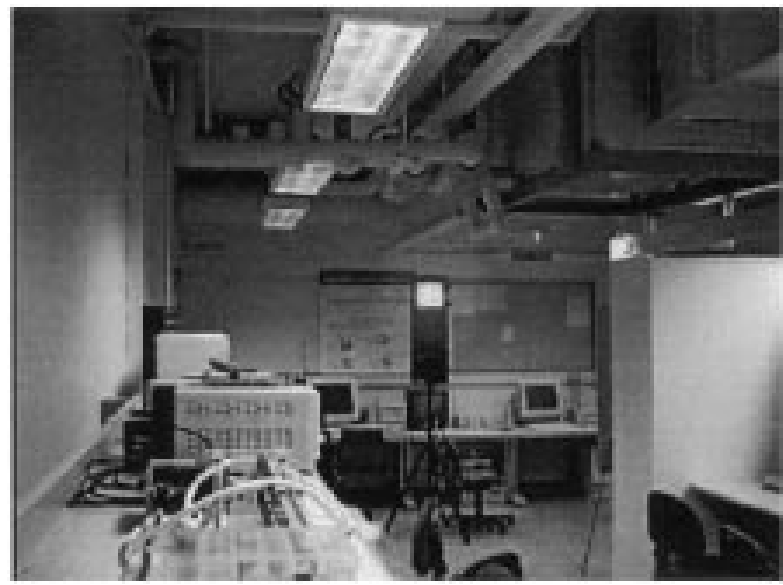

Fig. 34. Image with the LED Traffic light turned all on and shifted 2 pixel to the right.

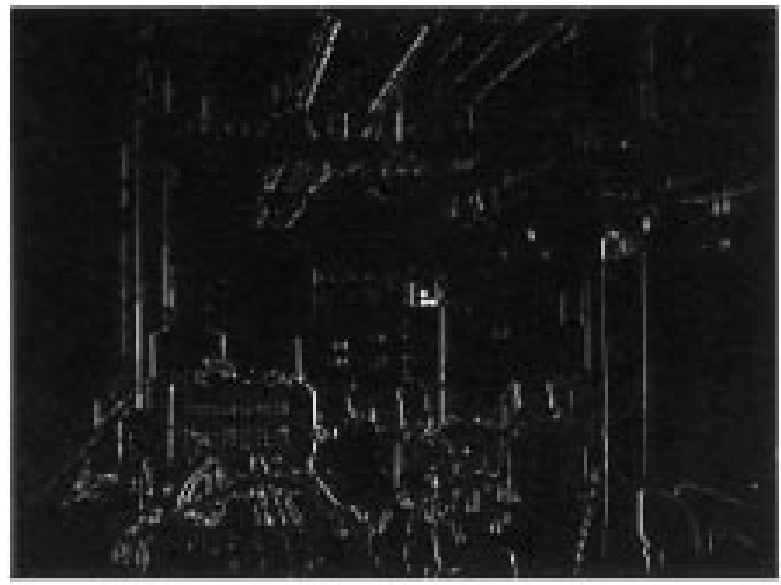

Fig. 35. A differenced image of Figs. 33 and 34 showing the complement $\left(\mathrm{X}^{\prime}\right)$ of pattern $\mathrm{X}$ with noises.

Thresholding is useful for removing noise with smaller intensity than the LED signal in the differenced image. Experimental results show that it can reduce much noise in the background and makes the LED signal clearer. Median filter is effective for removing small differencing noise as shown in the results. If the median filter radius is set too high, then the LED signal may be

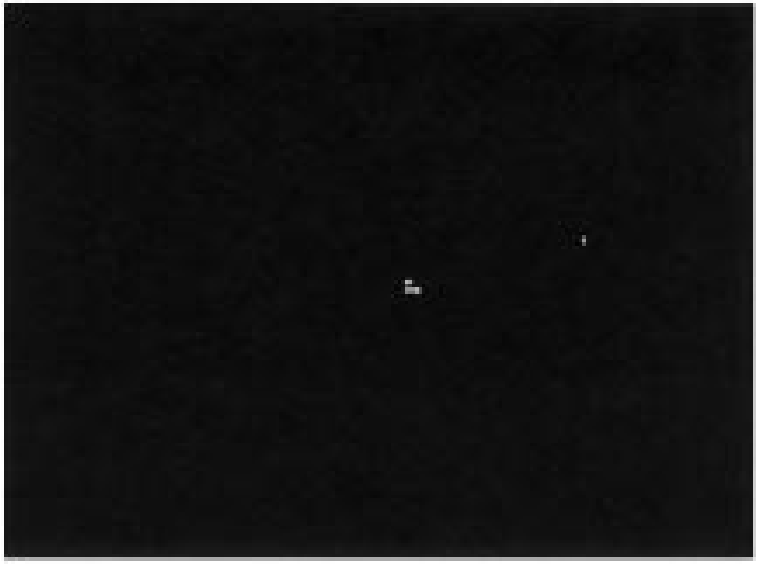

Fig. 36. Image after applying a thresholding process of level 160 (of 256) and three times of median filtering with radius of one pixel to Fig. 35

removed together with the noise. The processing time will also be longer.

\section{CONCLUSION}

The idea of a location beacon system based on a LED transmitter and a digital camera based receiver are developed and presented in this paper. These ideas and designs are original which can be an alternative to the existing location beacon system for vehicle positioning and related applications. A digital camera is used to capture images of an intelligent LED traffic light. The captured digital images are processed by the algorithm developed and a location code is extracted. The design issues of the system include the transmitter and the receiver structure, the signaling method, the transmission protocol, the required camera capturing rate and pattern update rate of the LED panel have been discussed.

Experiments using a prototype transmitter and a prototype receiver were performed which gave a good demonstration of the viability of the ideas and methodologies developed. The performance of the system depends on the structure of the LED panel, the resolution of the camera, the exposure technology of the camera, the speed of the image processor for real-time application and the movement of the camera and the surrounding objects. Further works include the use of an embedded image processing system as a beacon receiver, a higher frame rate camera, and intelligent algorithm for image processing [14].

The methodologies developed blend several hot technologies, and that includes the active pixel CMOS vision sensor, the high brightness LEDs, and digital image processing technique. The combination of these high technologies brings a new kind of communication means for the location beacon system in the field on ITS.

\section{REFERENCES}

[1] J. O'Connell, “The Philadelphia story," in Traffic Technol. Int., Aug./Sept.'97, pp. 106-110.

[2] G. Pang, K. L. Ho, T. Kwan, and E. Yang, "Visible light communication for audio systems," IEEE Trans. Consumer Electron., vol. 45, pp. $1112-1118$, Nov. 1999.

[3] G. Pang, H. Liu, C. Chan, and T. Kwan, "Vehicle location and navigation systems based on LEDs," in Proc. 5th World Congr. Intelligent Transport Systems, Seoul, Korea, Oct. 1998, pp. 12-16. 
[4] G. Pang, T. Kwan, C. Chan, and H. Liu, "LED traffic lights as a communication device," in Proc. Int. Conf. Intelligent Transportation Systems, Tokyo, Japan, Oct. 5-8, 1999, pp. 788-793.

[5] G. Pang, C. Chan, H. Liu, and T. Kwan, "Dual use of LEDs: Signaling and communications in ITS," in Proc. 5th World Congr. Intelligent Transport Systems, Seoul, Korea, Oct. 12-16, 1998.

[6] G. Pang, T. Kwan, H. Liu, and C. Chan, "Optical wireless based on high brightness visible LEDs," in Proc. IEEE Industry Applications Society Аnnu. Meeting, pp. 1693-1699, Oct. 3-7, 1999.

[7] G. Pang, C. Chan, and T. Kwan, "Tricolor light emitting dot matrix display system with audio output," IEEE Trans. Ind. Applicat., vol. 37, pp. 534-540, Mar./Apr. 2001.

[8] J. M. Kahn and J. R. Barry, "Wireless infrared communications," in Proc. IEEE, vol. 85, pp. 265-298, Feb. 1997.

[9] P. P. Smyth, P. L. Eardley, K. T. Dalton, D. R. Wisely, P. McKee, and D. Wood, "Optical wireless-A progonsis," in Proc. SPIE, vol. 2601, 1995, pp. 212-225.

[10] J. M. Kahn, R. You, P. Djahani, A. G. Weisbin, B. K. Teik, and A. Tang, "Imaging diversity receivers for high-speed infrared wireless communications," IEEE Commun. Mag., pp. 88-94, Dec. 1998.

[11] D. Ibrahim and M. Beasley, "The benefits of LED traffic lights in London and the pilot test sites," in IEE Conf. Road Transport Information and Control, Apr. 21-23, 1998, pp. 172-176.

[12] Dialight Light Emitting Diode LED Traffic Signal Specifications. (1999). [Online]. Available: http://www.dialight.com

[13] OV5017 Version 1.6 OmniVision Technologies, Inc. (1999). [Online]. Available: http://www.ovt.com

[14] H. S. Liu, "Integrated vehicle positioning system using sensors and image processing of beacon signal," M.Phil. Thesis, Univ. of Hong Kong, Hong Kong, 2000.

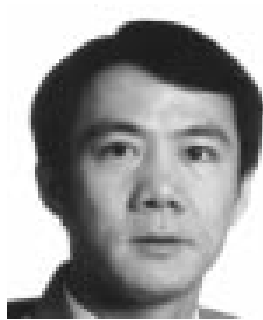

Grantham K. H. Pang (S'84-M'86-SM'01) received the Ph.D. degree from the University of Cambridge, Cambridge, U.K., in 1986.

From 1986 to 1996, he was with the Department of Electrical and Computer Engineering, University of Waterloo, ON, Canada. In 1994, he worked as a senior visiting researcher at Hitachi Research Laboratory, Hitachi-shi, Japan. In 1996, he joined the Department of Electrical and Electronic Engineering at The University of Hong Kong, Hong Kong. Since 1988, he published more than 120 technical papers and has authored and co-authored three books. His research interests include expert systems for control system design, intelligent control, intelligent transportation system, neural networks, control theory and computer-aided design.

Dr. Pang was awarded the ICI Prize, in 1989, for authorship of the best paper on the application of the theory of control published in the Transaction of the Institute of Measurement and Control. In 1996, he was the Organizing Chair of the IEEE Symposium on Computer-Aided Control System Design. From 1993 to 1995, he was the Chair of the Technical Committee on Computer-Aided Control System Design. He is an editor of the International Journal of Intelligent Control and Systems and the Journal of Control and Computers, published by the International Association of Science and Technology for Development (IASTED). $\mathrm{He}$ is a Chartered Electrical Engineer, and a member of the Institution of Electrical Engineers and the Hong Kong Institution of Engineers.

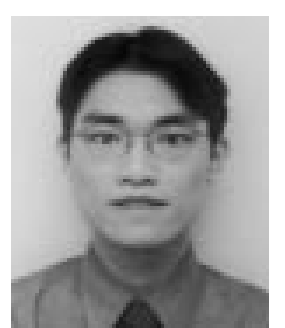

Hugh H. S. Liu (S'95-M'99) received the B.Eng. degree in 1997 and the M.Phil. degree in 2000, both from the Department of Electrical and Electronic Engineering of The University of Hong Kong, Hong Kong.

$\mathrm{He}$ is a Design Engineer in the Industrial Automation Laboratory in The University of Hong Kong, Hong Kong. His research interests include vehicle positioning sensors and systems, intelligent transportation system, vision systems and circuits, and image processing. 